\title{
Un rêve qui devient réalité
}

Jean Pouget

La Journée de Recherche Clinique «Innovation thérapeutique et parcours de soins » du 14 septembre 2018 a été organisée par l'AFM-Téléthon en partenariat avec la filière de santé maladies rares Filnemus. C'est le troisième partenariat du genre. En 2009 déjà, nous avions collaboré à l'organisation d'une Journée de Recherche Clinique sur les recommandations de prise en charge dans les pathologies neuromusculaires les plus fréquentes. Ces guidelines étaient les prémices des Protocoles Nationaux de Diagnostic et de Soins d'aujourd'hui, dont nous espérons vivement que plusieurs paraîtront d'ici peu. En 2013, l'AFM-Téléthon et Filnemus ont de nouveau collaboré à une Journée de Réflexion Clinique consacrée cette fois aux urgences. Elle a également donné lieu à des recommandations importantes, en particulier pour les équipes des services d'urgences et les sociétés savantes d'urgentistes. Le thème de ce nouvel évènement partagé - l'innovation thérapeutique - symbolise le passage d'un rêve à une réalité. Le traitement des maladies neuromusculaires, les personnes malades en ont rêvé. Leurs médecins également. Nous en sommes au stade où ce rêve devient une réalité tangible, fruit de beaucoup d'efforts. Notre impatience ne doit pas nous faire méconnaître certaines difficultés. La première d'entre elles porte sur la méthodologie des essais thérapeutiques. Le critère d'évaluation principal d'un essai, si important, n'est jamais parfait et ne fait pas toujours l'objet d'un consensus. Les maladies neuromusculaires sont rares, une caractéristique problématique pour évaluer un candidat médicament. Ce sont aussi des pathologies évolutives au long cours. Or la durée d'un essai thérapeutique est relativement courte. Dès lors, il ne nous fournit qu'un reflet très partiel de l'efficacité du nouveau traitement, mais également de sa tolérance. La phase des essais doit donc être préparée avec beaucoup de soins. Une autre difficulté méthodologique tient à la variabilité des formes génétiques des pathologies neuromusculaires, à l'exemple de l'amyotrophie spinale proximale lié au gène $S M N 1$. Doit-on considérer qu'un traitement efficace dans une forme de la maladie l'est obligatoirement dans une autre de ses formes, moins évolutive?

Les essais cliniques terminés, intégrer les innovations thérapeutiques au cœur du parcours de soins représente une activité supplémentaire et demande des compétences particulières, qu'il faut parfois acquérir. L'inertie de nos

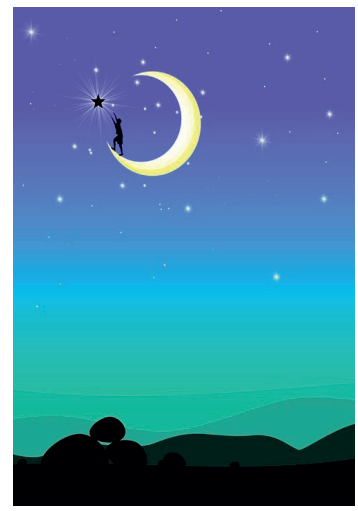

Coordinateur de la filière de santé maladies rares neuromusculaires (Filnemus). Assistance Publique-Hôpitaux de Marseille (APHM),

80 , rue Brochier, 13005 Marseille, France.

jpouget@ap-hm.fr

structures de soins ne nous facilite pas la tâche. II nous faut également composer avec des contraintes médico-économiques majeures. Une véritable innovation a un coût de développement important, que l'industrie pharmaceutique reporte volontiers sur le prix du médicament. Nous savons l'enveloppe des dépenses de santé bien définie. Des problèmes de choix vont se poser. Ce qui est donné à certains ne sera pas donné à d'autres. Notre société n'est pas encore habituée à opérer de tels choix, qui nécessitent un courage politique. Nos voisins britanniques ont été beaucoup critiqués pour avoir fait des choix de société parfois critiquables, mais courageux. Nous devons à notre tour les aborder et en débattre.

Passer du rêve à la réalité n'est pas facile. Le propre de l'activité onirique, c'est l'irréalisme. Nous sommes désormais entrés dans une phase de réveil, un peu brutale. Elle nous confronte aux problématiques budgétaires et d'organisation des soins liées à l'innovation thérapeutique. Tous ces aspects doivent être mieux anticipés. Ils passent bien entendu après le résultat : traiter le patient. De mauvais esprits diront que les cliniciens des maladies neuromusculaires vont arrêter leur vie contemplative pour enfin s'intéresser aux essais thérapeutiques et aux traitements innovants. Je n'en fais pas partie. Nous devons réagir de manière positive et avoir conscience de la grande chance que représente l'arrivée des thérapies innovantes. Les cliniciens vont devoir se retrousser les manches, avec enthousiasme. Ils savent le faire. Promouvoir l'accès aux traitements fait partie des ambitions phares du troisième Plan national maladies rares, qui prévoit une série d'actions dédiées. A nous de contribuer à les rendre effectives. $\diamond$

A dream becoming reality

\section{LIENS D'INTÉRÊT}

L'auteur déclare n'avoir aucun lien d'intérêt concernant les données publiées dans cet article. 